\title{
A New Segmentation Approach for Old Fractured Pieces
}

\author{
Jesus Llanes ${ }^{1}$, Antonio Adan ${ }^{1}$, and Santiago Salamanca ${ }^{2}$ \\ ${ }^{1}$ Escuela Superior de Informática. Universidad de Castilla-La Mancha, \\ 13071 Ciudad Real, Spain \\ \{Jesus.Llanes, Antonio. Adan\}@uclm.es \\ ${ }^{2}$ Escuela de Ingenierías Industriales. Universidad de Extremadura, \\ 06006 Badajoz, Spain \\ ssalaman@unex.es
}

\begin{abstract}
In this paper we propose a method for characterizing the surface of fractured pieces which come from old and complete original objects. Natural fractured pieces are difficult to segment due to the fact that the faces and edges are not well defined. For this reason, standard local feature based approaches are clearly inefficient to make an efficient segmentation. Our segmentation procedure is based on the Cone Curvature (CC) concept applied over the original dense models which provide standard scanner modeling tools. This CC basedmethod allows us to explore the surface from multiple neighborhood levels and to find a compact segmentation solution which characterizes different parts of the piece. A wide experimentation has been carried out on a set of old fractured pieces belonging to the remains of roman sculptures.
\end{abstract}

Keywords: 3D segmentation, 3D shape analysis, 3D data processing.

\section{Introduction}

The automatic reconstruction of fragmented objects through the matching of its fragments is a very common problem in archaeology, paleontology and art restoration. This is a challenging problem which has not been completely solved yet. It is possible to manually reconstruct; however, it may be a very tedious and difficult task for humans. For this reason, it is crucial to find methods that allow executing these tasks by help of a computer. The computer should select correct combinations of fragments on its own and yield coherent reconstructions.

Medium and high level 3D data processing (segmentation, labeling, recognition and understanding) over old fractured pieces is a very difficult field that remains currently under research. This is due to the fact that the original geometry has changed over ages without following a specific manipulation pattern turning into non-usual geometry. Therefore, most of conventional techniques applied on standard models are inefficient in this environment. Figure 1 shows a set of old pieces which we are currently working with.

The segmentation of the fragments into different faces and boundaries is one of the key steps when a reconstruction is carried out. In this area, many authors have proposed segmentation algorithms which work with usual objects ([1], [2], [3]). Most techniques are based on edge detection, region growing and probabilistic methods. 

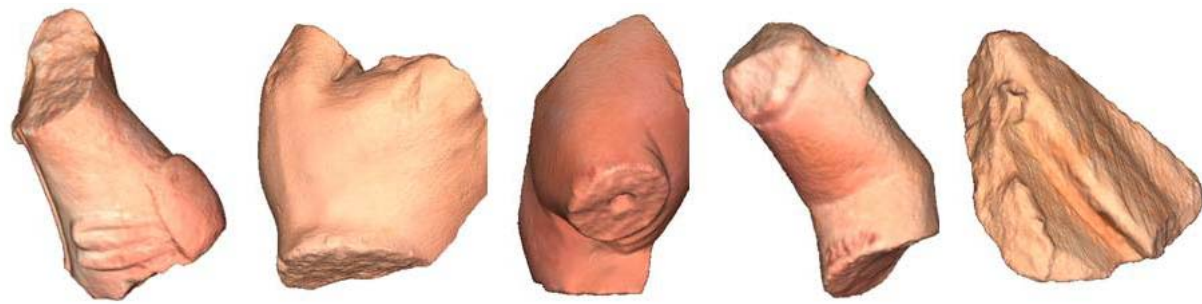

Fig. 1. Tridimensional models corresponding to a set of old fragments belonging to the Roman Art Museum of Merida (Spain)

Edge detection techniques are those which allow detecting the edges of an object to segment it in faces. Several representative works follow.

Liu [4] proposed a method for 3D surface segmentation algorithm based on the classical Robert's operator into 3D space, which works by computing the sum of the squares of the differences between diagonally adjacent pixels. Zucker and Hummel [5], developed an algorithm to perform an optimal three dimensional edge detection based on a Sobel operator which extract the edges using the magnitude of the image gradient at each node of the object.

On the other hand Huang et al [6] perform the segmentation of the object into faces using a multi - scale edge extraction. The algorithm that implements the multi - scale edge extraction is based on the curvature of the surface, measured by the integral invariants [7]. Bellon and Silva [8] perform an object segmentation method based on the edge extraction. The algorithm detects de edge points by the comparison between the normal angles of the surface points of the object. Gotardo et al. [9] proposes a segmentation method which classifies the surfaces points as flat or curved. To make this the algorithm calculates the angular change in the normal direction in moving from a point to nearby points.

Region growing techniques are based on extracting a region of an object using some predefined criteria. From this seed point, the algorithm grows finding connecting areas that fit to the predefined criteria.

In Papaioannou et al [10], [11], [12], segmentation is performed by a region growing method based on the average normal of the polygons that form the mesh. The procedure begins with an arbitrary polygon. The adjacent polygons are classified as related to the same face if their average normal do not deviate more than a predefined threshold.

Generally, the method used to segment archaeological objects is based on edges detection. However, it is very difficult to detect the edges due to the fact that some of the fragments have very smooth borders because of erosion. In Figure 2a) some fragments belonging to a set of archaeological finds are shown. The smooth segments of the borders are marked. When an edge detection algorithm is applied to an object that has smooth borders, it is not impossible to detect them correctly. We applied an algorithm to detect the edges based on the simple curvature values of the mesh nodes. The edge nodes detected by this algorithm are shown in Figure $2 \mathrm{c}$ ). It could be noted that the algorithm did not detect any point in the segment of the border that is especially smooth. If we try to define a region delimited by the extracted edges, the system fails 


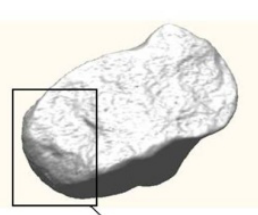

(a)

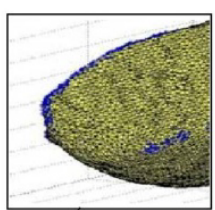

(c)
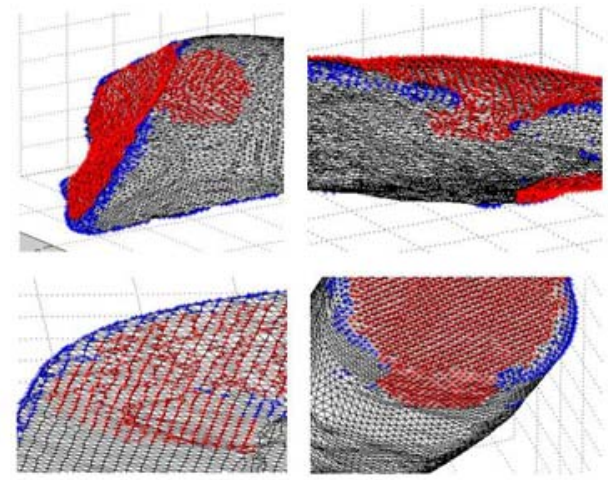

d)

Fig. 2. a) View of the whole object. b) Partial view of the edge segment especially smooth. c) Edge nodes detected by a conventional algorithm. d) Incorrect segmented faces due to the inappropriate detection of the edges.

and usually we could note that the region continues growing, exceeding their real contours. In Figure 2 d) we show segmented faces that are clearly incorrect due to an inappropriate detection of the edges.

The results obtained in the experiments with archaeological objects demonstrate that it is very difficult to implement an effective segmentation algorithm based on edge detection techniques over conventional criteria (i.e. Gaussian curvature). For this reason, it is necessary to use a more robust method.

\section{Segmentation Algorithm}

In this section we present a segmentation algorithm which combines edge detection based on CC, edge cycling paths obtaining and region growing. Since Cone Curvature is the geometrical feature we have used, a brief introduction about it follows.

\subsection{Cone Curvature Feature}

Cone curvature is a geometrical feature originally defined and applied on spherical meshes [13] and lately used for clustering purposes in triangular meshes [14].

Let $\mathrm{M}_{\mathrm{T}}$ be a triangular mesh fitted to an object and let $\mathrm{N}$ be a vertex of $\mathrm{M}_{\mathrm{T}}$. Note that $\mathrm{M}_{\mathrm{T}}$ has been previously regularized and resampled to a fixed number of nodes $h$. We organize the rest of the nodes of the mesh in concentric groups spatially disposed around N. Since this organization resembles the shape of a wave it is called Modeling Wave $(M W)$. Consequently, each of the disjointed subsets is known as Wave Front (WF) and the initial node $\mathrm{N}$ is called Focus. Let us call all the possible MWs that can be generated over T Modeling Wave Set (MWS). Figure 3 a) illustrates the mesh model of an object and several wave fronts plotted for two different focuses.

Cone Curvature is defined taking into account MWS structure. We define Cone Curvature $\alpha^{j}$ of $\mathrm{N} \in \mathrm{M}_{\mathrm{T}}$, the angle of the cone with vertex $\mathrm{N}$ whose surface inscribes the jth Wave Front of the Modeling Wave associated to N. 


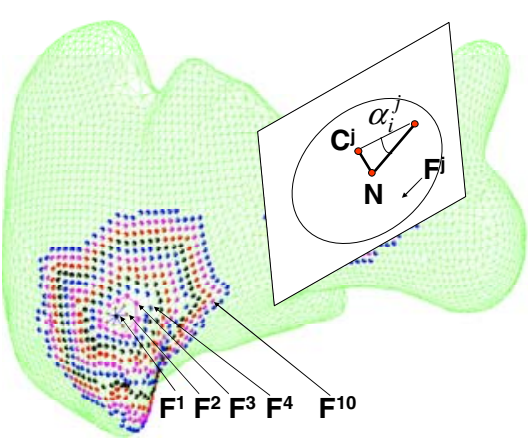

a)
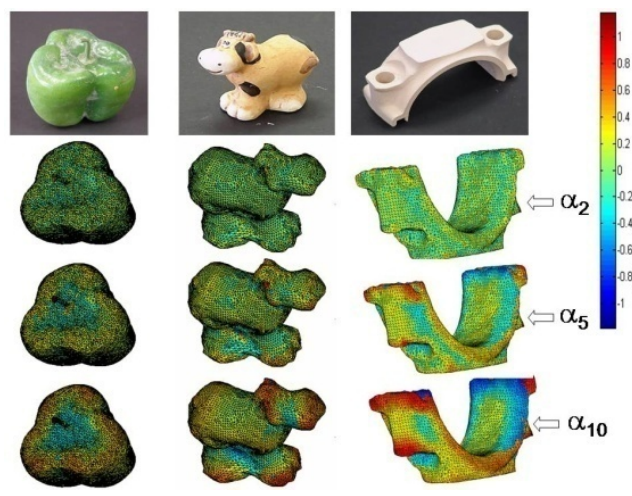

b)

Fig. 3. a) Wave fronts in two different focuses printed over the mesh model and representation of $\mathrm{j}$-th cone curvature in a point of the object. b) Illustration of several CC orders.

The range of $\mathrm{CC}$ values is $[-\pi / 2, \pi / 2]$, being the sign assigned taking into account the relative location $C^{j}$ with respect to $\mathrm{M}_{\mathrm{T}}, C^{j}$ being the barycentre of the $j$ th WF. Figure 2 right illustrates this definition.

Note that a set of values $\left\{\alpha^{l}, \alpha^{2}, \alpha^{3}, \ldots \alpha^{q}\right\}$ gives an extended curvature information around $\mathrm{N}$ until the $q$ th WF, where the word 'curvature' has a non-local meaning. So for each node $\mathrm{N}$ a set of $q$ values could be used for exploring its surroundings. From now on we will call them Cone Curvature Levels. Thus, as the ring increases a new $\mathrm{CC}$ level is defined. Thus the Cone Curvatures offer wider information about the surroundings of a point and are a very flexible descriptor because it is possible to select one or more $\mathrm{CC}$ levels according to the specifications of the shape to be analyzed. Figure $3 \mathrm{~b}$ ) illustrates the $\mathrm{CC}$ values for a set of orders following a color scale over geometrical models belonging to non-fractured objects.

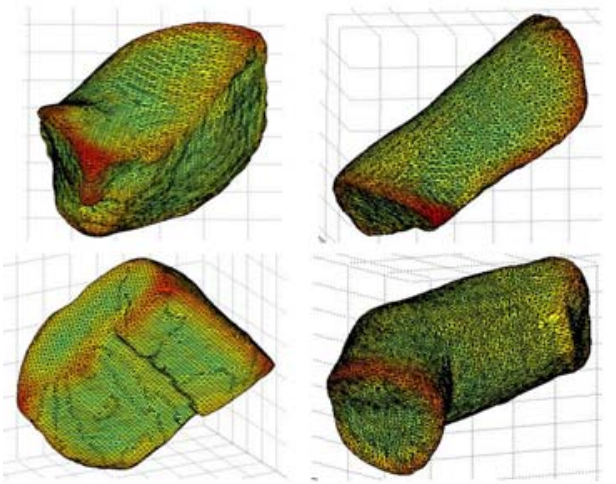

a)

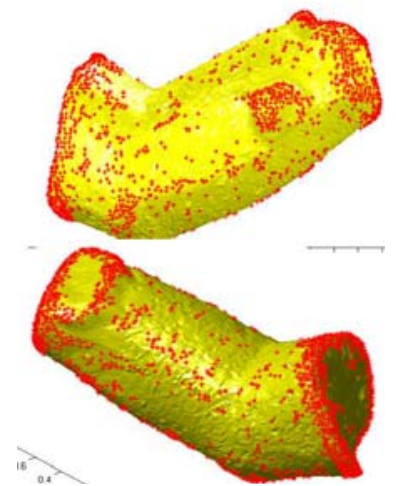

b)

Fig. 4. a) Objects represented with the cone curvature values of its nodes in a colored scale. b) Edge-nodes detected for low (level 4) and high (level 15) CC levels. 
In Figure 4 a) we also show a graphical representation of the $5^{\text {th }}$ Cone Curvature calculated on a set of fractured pieces. Note that the regions in red are those which have highest cone curvature values and the regions colored in green are those which have the smaller values. Figure $4 \mathrm{~b}$ ) presents edge-nodes for several CC values.

\subsection{Segmentation Using $\mathrm{CC}$}

In fractured objects the segmentation of the original triangular model $\left(\mathrm{M}_{\mathrm{T}}\right)$ taking into account only one specific CC level might not be achieved. Thus, to define the set of edges in the mesh and to find cycling-edges paths we need to test several CC levels as well as to turn the triangular mesh representation (used in conventional CAD tools and 3D scanner software) into a semi-regular mesh with controlled topology.

Note that if we take a low CC level, we will have discontinuous sets of edge-nodes whereas if we take high $\mathrm{CC}$ levels, we might obtain crowded edges. Firstly, in fractured pieces like the ones presented in Figure 1, there exist edges or part of edges which are sharp whereas others are smooth, probably due to erosion effects that the pieces suffer over time. Theoretically the sharp edges can be detected for low $\mathrm{CC}$ levels and the smooth ones for medium or, maybe, high CC levels. In practice it doesn't happen in all the cases. Secondly, in both cases using a triangular-patch mesh without a regular topology it is really hard, if not impossible, to find edge-paths in such a geometrical structure. Therefore, in both cases, the extraction of edges, the connection between edges and the search for cycling edge-paths will be inefficient.

In order to control the topological problem, a new model $\mathrm{M}_{\mathrm{E}}$ fitted to the object is used. The model $\mathrm{M}_{\mathrm{E}}$ has regular 3-connectivity with invariable number of nodes. This canonical model comes from the implosion of a tessellated and semi-regular sphere over the object. Figure 5 a) illustrates the transformation $\mathrm{M}_{\mathrm{T}}$ to $\mathrm{M}_{\mathrm{E}}$. This model corresponds to the initial Modeling Wave (MW) topology built on spherical representation models [11]. In the aforementioned topology a node is 3-connected with its neighbors but also is connected, in recursive manner, with the neighbors of the neighbors.

Assuming that the spherical model of the object has been generated, the segmentation algorithm is composed of the following stages.

1.- Calculate the cone curvature values on $\mathbf{M}_{T}$ for a minimum initial level. This value is imposed trough a lineal function taking into account the density of the mesh $\mathrm{M}_{\mathrm{T}}$.

2.- Define the set of edge-node candidates $G$ on $M_{T}$ by imposing a $C C$ threshold $\mu$. This threshold is calculated through the histogram of CC at the specified level.

3.- Map $\mathrm{G}$ into model $\mathrm{M}_{\mathrm{E}}$.

4.- Filter outlier edge-nodes. For instance outlier edge-nodes are nodes which have less than two edge-node neighbors.

5.- Find minimum closed-paths in $\mathrm{G}$ trough the controlled topology of $\mathrm{M}_{\mathrm{E}}$. The algorithm is based on a graph-theory recursive search. Generate cycling edge-paths

6.- If there exist open paths in $\mathrm{G}$ then take the next $\mathrm{CC}$ level an go to step 2

7.- Remap the edges into $\mathrm{M}_{\mathrm{T}}$.

8.- Take an initial random seed and apply a region growing algorithm on $\mathrm{M}_{\mathrm{T}}$.

9.- Save the segment and update the search regions.

10.- Go to 8 until there are no seeds. 
Note that, when we have obtained a correct detection of the edges and the cycling boundaries of the regions (step 8), we can employ a region growing algorithm to define the faces of the object. It is well known that region growing is one of the simplest region-based segmentation methods. The region growing method begins taking a random seed on $\mathrm{M}_{\mathrm{T}}$. We check the adjacent nodes and add them to the region if they are not marked as edge-node. Thus the region grows until all nodes of its boundary have some edge-node neighbor. Figure 5b) illustrates results in several steps of the segmentation algorithm.

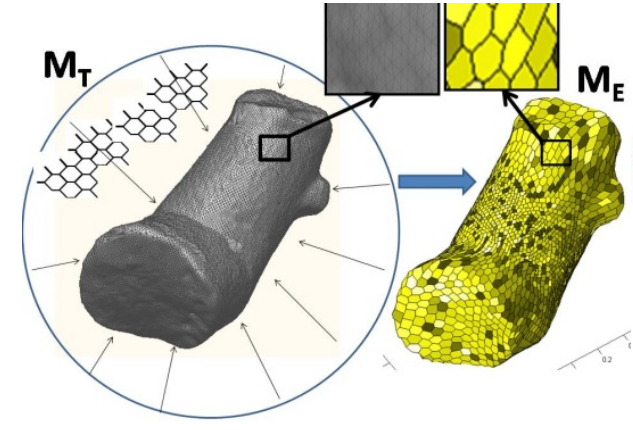

a)

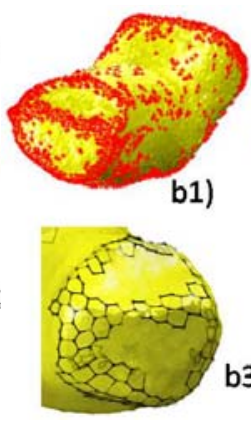

b3)

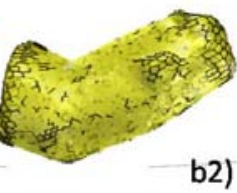

b2)

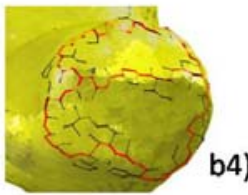

b)

Fig. 5. a) Model transformation. Generating model $M_{E}$ by implosion of a spherical tessellated mesh into $\mathrm{M}_{\mathrm{T}}$. b1) The set of edge-node candidates on $\mathrm{M}_{\mathrm{T}}$. b2) Edge-node candidates mapped onto model $\mathrm{M}_{\mathrm{E}}$. b3) Result after filtering outlier edge-nodes, b4) Generating cycling paths in $\mathrm{M}_{\mathrm{E}}$ and remapping into $\mathrm{M}_{\mathrm{T}}$.

\section{Experiments and Future Work}

The fragments we are currently segmenting and labeling belong to old sculptures. Our lab, in collaboration with the National Museum of Roman Art of Mérida (Spain) research department, is currently working on a project concerning the digitalization and reconstruction of such sculptural groups dating to the first century B.C. One of the purposes of this project is to solve integration problems of the synthesized models of original pieces in a hypothetical virtual model of the whole sculptural group. In practice, a few real pieces are available. Then, a possible solution is based on, having a priori knowledge of the original sculpture, developing new surface correspondence techniques - which include heuristics and the aid of expert systems - which help to place each part in its original position. In this general proposal, segmentation and labelling of the faces of a fractured piece is a crucial task in making the registering algorithm efficient enough.

Figure 6 a) shows the work developed so far on a sculptural group where only 30 pieces have been recovered. It can be seen the integration of different fractured pieces in the virtual model. Three of them are big pieces that can be easily recognized as body parts. The rest of the pieces are smaller fragments and their identity and position in the original group is currently unknown. Some of these pieces and the results after 


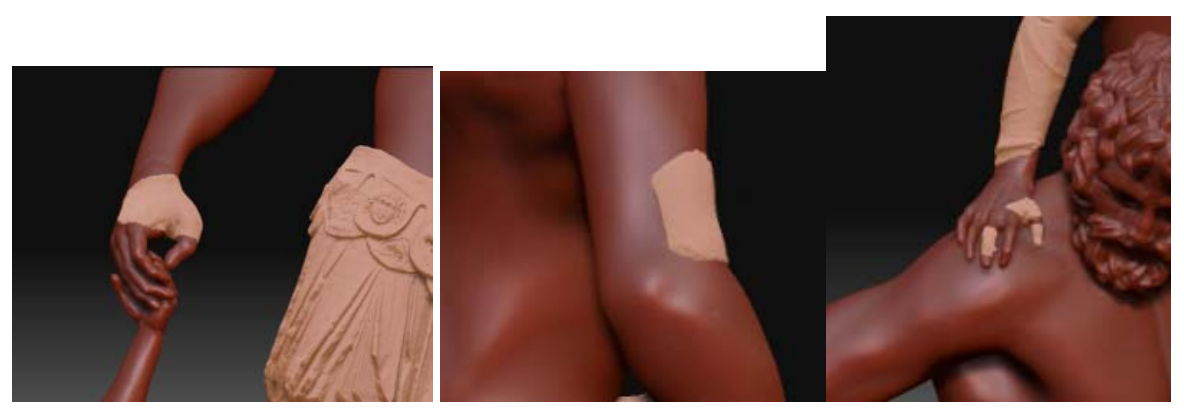

a)
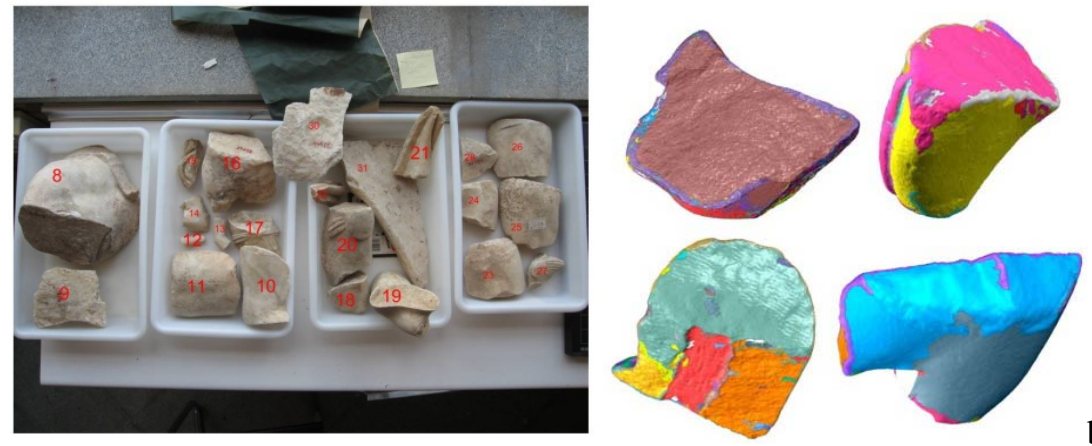

b)

Fig. 6. a) Coupling of several fragments into a virtual model. b)Set of segments belonging to a original sculpture and segmentation results.

segmentation can be seen in Figure $6 \mathrm{~b}$ ). Beard in mind that the segmented areas do not follows a rigid and usual pattern like in non-manipulated object cases. Here the face contours have irregular shapes. We are currently working on improving the segmentation and labeling of more complex pieces and cases. This is a very difficult problem which needs to be well defined and solved. Therefore, we aspire to solve the problems that we encountered on some of the previous related works. The presented solution should of course be improved and generalized for a wider variety of objects in the archaeological area.

Future works are addressed to develop efficient intelligent algorithms to help archaeologist to reconstruct incomplete 3D puzzles. In this sense, we aim to extend and improve the current semi-automatic solutions and provide an expert system based on fuzzy logic which is able to propose a limited number of solutions which can be evaluated by historians and archaeologist.

\section{Conclusions}

Standard local feature based approaches are clearly inefficient to make an efficient segmentation in old fractured pieces. This paper presents a new solution in this field by using edge detection algorithms based on the cone-curvature concept. CC allows us to explore the surface from multiple neighborhood levels and to find a compact 
segmentation solution which characterizes different parts of the piece. A wide experimentation has been carried out on several old pieces belonging to Spanish $\mathrm{Mu}$ seum of Roman Art yielding promising results.

\section{Acknowledgment}

This work is supported by DPI2006-14794-C02 and PCI08-0052-1401 projects and with the collaboration of the Spanish Museum of Roman Art.

\section{References}

1. Haralick, R.M., Shapiro, L.G.: Image Segmentation Techniques. Computer Vision, Graphics, and Image Processing (January 1985)

2. Pal, N.R., Pal, S.K.: A Review on Image Segmentation Techniques. Pattern Recognition (1993)

3. Liu, H.K.: Two and Three Dimensional Boundary Detection. Computer Graphics and Image Processing (April 1977)

4. Herman, G.T., Liu, H.K.: Dynamic Boundary Surface Detection. Computer Graphics and Image Processing (1978)

5. Zucker, S.W., Hummel, R.A.: An Optimal Three- dimensional Edge Operator. Technical report, McGil University, Toronto, Ontario, Canada (April 1979)

6. Huang, Q.-X.: Reassembling Fractured Objects by Geometric Matching. ACM Transactions on Graphics (TOG) archive 25(3) (July 2006)

7. Pottman, H., Wallner, J., Huang, Q.-X., Yang, Y.: Integral invariants for robust geometry processing. Computer Aided Geometric Design 26, 37-60 (2009)

8. Bellon, O., Silva, L.: New improvements to range image segmentation by edge detection. IEEE Signal Processing Letters 9(2), 43-45 (2002)

9. Gotardo, P., Bellon, O., Boyer, K., Silva, L.: Range Image Segmentation Into Planar and Quadric Surfaces Using an Improved Robust Estimator and Genetic Algorithm. IEEE Transactions on Systems, Man and Cybernetics. Part B, Cybernetics 34(6), 2303-2316 (2004)

10. Papaioannou, G., Karabassi, E.-A., Theoharis, T.: Virtual Archaeologist, Assembling the Past. IEEE, Computer Graphics and Applications 21, 53-59 (2001)

11. Papaioannou, G., Karabassi, E.-A., Theoharis, T.: Reconstruction of three-dimensional objects through matching of their parts. IEEE Transactions on Pattern Analysis and Machine Intelligence 24(1), 114-124 (2002)

12. Papaioannou, G., Karabassi, E.-A.: Automatic Reconstruction of Archaeological Finds - A Graphics Approach. IEEE Transactions on Pattern Analysis and Machine Intelligence 24(1), 114-124 (2002)

13. Adán, A., Adán, M.: A Flexible Similarity Measure for 3D Shapes Recognition. IEEE Transactions on Pattern Analysis and Machine Inteligence 26(11) (November 2004)

14. Adán, A., Adán, M., Salamanca, S., Merchán, P.: Using Non Local Features For 3D Shape Grouping. In: da Vitoria Lobo, N., Kasparis, T., Roli, F., Kwok, J.T., Georgiopoulos, M., Anagnostopoulos, G.C., Loog, M. (eds.) SSSPR 2008. LNCS, vol. 5342, pp. 644-653. Springer, Heidelberg (2008) 